\title{
Topical Collection: Sleep apnea syndrome: from the pathogenesis to the last therapies
}

Special Guest Editors: Manuele Casale, Rinaldi Vittorio

(c) Springer-Verlag GmbH Germany, part of Springer Nature 2021 